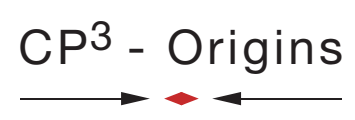

\title{
Nonperturbative Results for Yang-Mills Theories
}

\author{
Francesco SANNINO \\ - $C P^{3}$-Origins, Campusvej 55, DK-5230 Odense M, Denmark. \\ Joseph SCHECHTER ${ }^{2} †$ \\ * Dept. of Physics, Syracuse University, Syracuse, New York 13244-1130, USA
}

\begin{abstract}
Some non perturbative aspects of the pure SU(3) Yang-Mills theory are investigated assuming a specific form of the beta function, based on a recent modification by Ryttov and Sannino of the known one for supersymmetric gauge theories. The characteristic feature is a pole at a particular value of the coupling constant, $g$. First it is noted, using dimensional analysis, that physical quantities behave smoothly as one travels from one side of the pole to the other. Then it is argued that the form of the integrated beta function $g(\mu)$, where $\mu$ is the mass scale, determines the mass gap of the theory. Assuming the usual QCD value one finds it to be $1.67 \mathrm{GeV}$, which is in surprisingly good agreement with a quenched lattice calculation. A similar calculation is made for the supersymmetric Yang-Mills theory where the corresponding beta function is considered to be exact.

Preprint: $C P^{3}$-Origins-2010-36
\end{abstract}

\section{INTRODUCTION}

The exact beta function of a gauge theory is generally considered to contain many non-perturbative secrets of the gauge theory behavior. Unfortunately it seems to be only computable analytically in perturbation theory. Physically it is related to the trace anomaly, or the non-zero value of the divergence of the scale (dilation) current. We are specializing to massless theories here so it represents a violation of the classical result.

Another object in gauge theories with massless fermions, the divergence of the $\mathrm{U}(1)$ axial vector current, should be zero in the classical limit but is known not to vanish at the quantum level (axial anomaly). Most interestingly it has the property that there are no corrections [1] beyond the one loop level so it can be considered to be known exactly.

Now, in supersymmetric gauge theories, it was found [2] that both the trace anomaly and the axial anomaly (actually together with the divergence of a special superconformal current) belong to the same chiral multiplet and hence should be somehow related to each other. There was a lot of discussion about the meaning of this feature - for example is there a contradiction between an exact one loop result for the axial anomaly and a result containing all orders of coupling constant for the trace anomaly - and finally it was realized that there could be compatibility, with the exact beta function being determined from the axial anomaly. This was shown both for the supersymmetric Yang-Mills theory [3] and for the supersymmetric theory containing also fermions not belonging to the adjoint representation [4]. A characteristic feature of these exact beta functions is a pole at a particular value of coupling constant, $g$ in the beta function

${ }^{*}$ Electronic address: sannino@cp3.sdu.dk

${ }^{\dagger}$ Electronic address: schechte@phy.syr.edu $\beta(g)$.

Recently, Ryttov and Sannino [5] conjectured such all order beta functions for ordinary $S U(N)$ (non supersymmetric) gauge theories both with and without fermions belonging to arbitrary representations based on analogy to the supersymmetric case. The generalization, by one of the authors, for the $S O$, and $S p$ gauge groups appeared in [6] and for chiral gauge theories in [7]. These beta functions were found to satisfy known consistency conditions at second order and to work well in many interesting applications to working technicolor models reviewed in[7]. These beta functions also feature a pole at a particular value of $g$.

In the present note, we study some non-pertubative consequences of the conjectured beta function for the simple SU(N) Yang-Mills theory; already [5] the conjectured beta function had been seen to give a reasonable picture in the asymptotically free perturbative region. In section II we use dimensional analysis to investigate the running with scale of physical quantities with various engineering dimensions. This involves $g(\mu)$ but it was noted that physical quantities change smoothly as g goes through the pole value.

In section III, we investigate the integration of the defining equation for the beta function which yields an explicit expression for $g(\mu)$. We consider all Yang-Mills theories in the sense that a full range of values for the coupling constant at a reference mass are considered. An amusing feature is seen to arise: the solutions for $g(\mu)$ do not allow $\mu$ to be lower than a certain value. We interpret this as the measure of the mass gap for the Yang-Mills theory. For the usual value of the QCD coupling constant, with $\mathrm{N}=3$, our predicted value of 1.67 $\mathrm{GeV}$ is seen to be in good agreement with a valence lattice calculation. We also compute the mass gap for the supersymmetric $\mathrm{SU}(3)$ and $\mathrm{SU}(\mathrm{N})$ Yang-Mills theories. In the latter case, the starting beta function does not require any conjecture.

Section IV contains a brief summary and discussion. 


\section{GENERAL SCALING RESULTS FOR YANG - MILLS THEORIES}

Consider a renormalization group invariant quantity $H$ of mass dimension $D$ in any theory with a single field and a single coupling constant $g$. Define $\mu$ to be the renormalization scale and $g(\mu)$ to be the value assumed by the coupling constant at that scale. Since $H$ must be a measurable physical quantity, dimensional analysis implies that it has the form,

$$
H=\mu^{D} \mathcal{H}[g(\mu)],
$$

where $\mathcal{H}[g(\mu)]$ is some function of the coupling constant. Of course, for any given theory there are many different interesting quantities $H$. One may, as usual, introduce a characteristic invariant scale, $\Lambda$ for the theory by defining a particular $H$ to be $\Lambda^{D}$. By differentiating both sides of Eq. 11) with respect to $\log (\mu)$, the natural $\log$, and recognizing that the left hand side is independent of $\mu$, we obtain the main equation:

$$
D \mathcal{H}+\frac{\partial \mathcal{H}}{\partial g} \beta(g)=0, \quad \text { with } \quad \beta(g)=\frac{\partial g}{\partial \log (\mu)} .
$$

By integrating this equation one immediately finds:

$$
\log \left(\frac{\mathcal{H}}{\mathcal{H}_{0}}\right)=-D \int_{g_{0}}^{g} \frac{d g}{\beta(g)} .
$$

Hence if the exact beta function were known as a function of $g$, one could express any renormalization group invariant quantity $H$ as a known function of $g$ up to an arbitrary overall constant.

Now, as discussed in the preceding section, a conjectured all orders beta function for the ordinary Yang-Mills theory based on the known supersymmetric Yang-Mills theory all orders beta function was recently introduced in [5] by Ryttov and Sannino. It was found to be consistent with other non perturbative approaches to the ordinary (non supersymmetric) gauge theories and hence to be a reasonable model for further investigation.

This RS all orders beta function ansatz for the SU(N) Yang-Mills theory reads:

$\beta_{Y M}=-g^{3} \frac{a}{1-b g^{2}}, \quad$ with $a=\frac{11}{3} \frac{N}{(4 \pi)^{2}} \quad$ and $b=\frac{17}{11} \frac{N}{8 \pi^{2}}$.

Using Eq. (4) in Eq.(3) then yields:

$$
H=\operatorname{const} \frac{\mu^{D}}{g^{\frac{D b}{a}}} \exp \left[-\frac{D}{2 a g^{2}}\right],
$$

where the overall constant is defined by

$$
\text { const }=\mathcal{H}_{0 g_{0}}{ }^{\frac{D b}{a}} \exp \left[\frac{D}{2 a g_{0}^{2}}\right] \text {. }
$$

Here the subscript zero denotes the value corresponding to the lower limit of integration in Eq. (3). Of course, there is a different numerical constant for each choice of $\mathrm{H}$. These numerical constants might be approximated by using perturbation theory at a large value of $\mu$, for example.

For definiteness we list some possible interesting choices for $H$.

i. The gluon condensate or vacuum expectation value of the trace of the energy momentum tensor. This has the engineering dimension $D=4$.

ii. The glueball squared masses $(D=2)$. We presume that there is a spectrum of glueballs with different spin-parities and masses.

iii. The glueball-glueball scattering cross sections ( $D$ $=-2)$.

iv. The coefficients, $a_{n}$ in the expansion of the partial wave amplitudes for glueball scattering, $\Sigma a_{n} s^{n}$ in a region of analyticity (Here $D=-2 n$ ).

To get an idea of the dependences of various physical quantities on the coupling constant $g$ we plot the characteristic factor,

$$
F(g)=\frac{1}{g^{\frac{D b}{a}}} \exp \left[-\frac{D}{2 a g^{2}}\right]
$$

in Eq.(5) for the case $D=4$ in Fig. 11(a) one and for the case $D=-2$ in Fig. 11(b) . For definiteness the choice $N$ equal to 3 is made. It is seen that each of these curves displays an extremum at the same value of $g$.

Differentiating shows that this value is,

$$
g^{2}=1 / b=\frac{88 \pi^{2}}{51} \approx(4.127)^{2} .
$$

Furthermore, the second derivative of $F(g)$ at this point is simply $-2 D F(g) / \mathrm{ag}^{4}$; this means that dynamical quantities with positive dimension $D$ will have a maximum at this point while those with negative $\mathrm{D}$ will have a minimum at the same point. We also note the interesting fact that for a given value of the physical quantity $F$, there are two different values of $g$. The situation will be further explored by looking at the beta function.

\section{BETA FUNCTION AND MASS GAP}

A graph of the $N=3$ Yang-Mills conjectured beta function in Eq. (4) is shown in Fig. 2(a). From its shape one concludes that the origin, $g=0$ is an ultraviolet stable fixed point and that there is clearly a pole at $g=b^{-1 / 2}$. However, we observe from the Figs. 1 (a) and (b) that the physical quantities, $H$ remain smooth as one goes from one side of the pole to the other.

In order to discuss the coupling constant in a gauge theory, one chooses a reference value $\mu_{0}$ of the energy scale and specifies (from experiment) the value of the 




(a) Case $D=4$

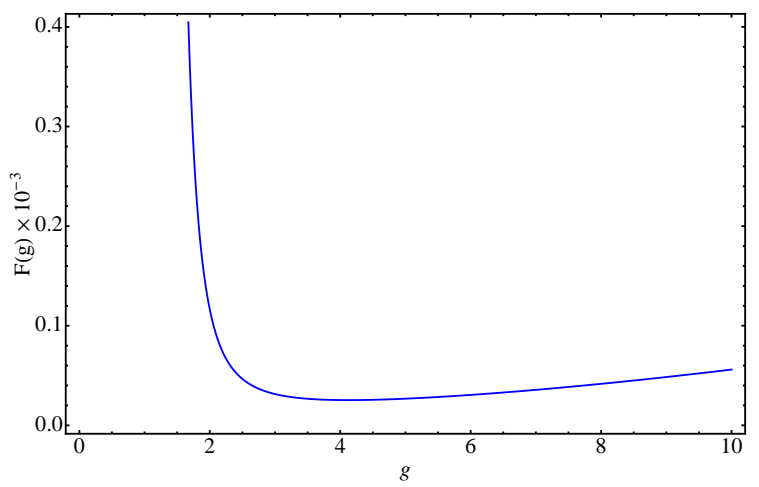

(b) Case $D=-2$

FIG. 1: $F(g)$ as function of $g$ for $N=3$.

running coupling constant, $g\left(\mu_{0}\right)$ at this point. In the case of QCD, it is convenient to choose $\mu_{0}=m_{Z} \approx 91.19$ $\mathrm{GeV}$. For illustration we will also choose this value of $\mu_{0}$ as our reference scale. The value of the QCD coupling constant at this scale is measured to be about 1.228. In the present pure Yang-Mills case we are dealing with a hypothetical model so we are free to choose any value for $g\left(\mu_{0}\right)$. In fact it is better to allow $g\left(\mu_{0}\right)$ to range over all possible values, which corresponds to describing all pure Yang-Mills $\mathrm{N}=3$ theories.

Clearly, the first step is finding out how the coupling constant runs with the scale $\mu$. Integrating the second of Eq. (2) yields the following relation between $g=g(\mu)$ and $\log (\mu)$ corresponding to any choice of $g_{0}=g\left(\mu_{0}\right)$ :

$$
\log \left(\frac{\mu}{\mu_{0}}\right)=\frac{1}{2 a}\left(\frac{1}{g^{2}}-\frac{1}{g_{0}^{2}}\right)+\frac{b}{a} \log \left(\frac{g}{g_{0}}\right)
$$

It is convenient to plot in Fig. 2(b) $\log \left(\mu / \mu_{0}\right)$ as a function of $g$ with the choice $g_{0}=1$. In the part of this plot to the left of $g(\mu)=b^{-0.5} \approx 4.127, g(\mu)$ decreases as $\log (\mu)$ increases. That is the expected asymptotically free behavior. On the other hand, to the right of this point, $g(\mu)$ smoothly starts rising with increasing $\log (\mu)$. There is no discontinuity at the pole. Clearly, this behavior is the same as that shown for physical quantities in panels

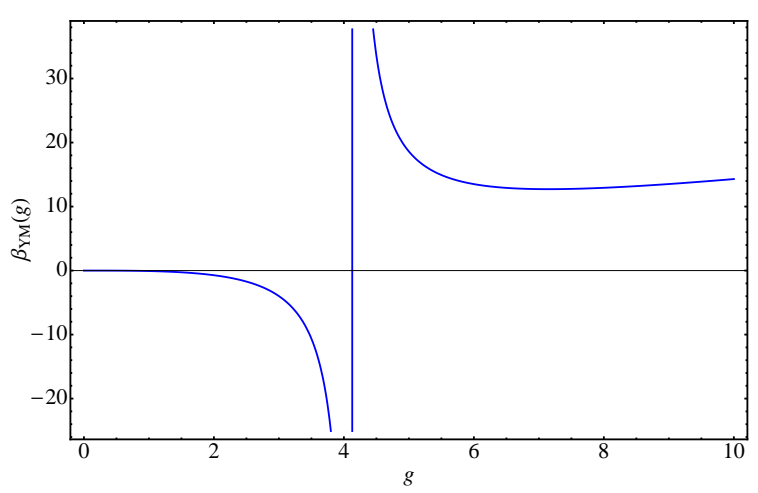

(a) $\beta_{Y M}(g)$ as function of $g$ for $N=3$.



(b) $\log \left(\mu / \mu_{0}\right)$ as function of the coupling $g$

FIG. 2: Plots of the YM beta function, panel (a), and of the solution for the running of the scale (b) as function of the coupling constant $g$ with $g_{0}=1$.

(a) and (b) of Figs. 1. The existence of a smoothly connected different phase for the theory is intriguing. For the present, however, we will concentrate on the asymptotically free region.

The different $N=3$ pure Yang-Mills theory which is defined by $g_{0}=3$ yields the running coupling constant plotted in Fig. 3(a). The overall picture is substantially the same. In particular, the asymptotically free region still corresponds to $g(\mu)<b^{-0.5}$. Similarly the theory characterized by $g_{0}=0.5$ is seen from the plot in Fig. 3(b) to still have the same range in $g(\mu)$ for the asymptotically free region, but with rather different $\mu$ values.

Figures 2(b), 3(a) and 3(b) each illustrate that there is a particular (but different in each case) value of $\log (\mu)$ below which the curve does not extend. This is peculiar since it would imply that the running coupling constant could not be measured experimentally in that region. The only way in which this might be consistent is if the disallowed region would be lower than $M$, twice the mass of the lightest glueball state in the theory. Then it would be below threshold and not accessible to experiment. That seems like a plausible determination of the mass gap of the theory. It is clear that the value of such a mass gap is determined by the ordinate of the point where $\log (\mu)$ is minimum, i.e. where $g=b^{-0.5}$. Specifically, 


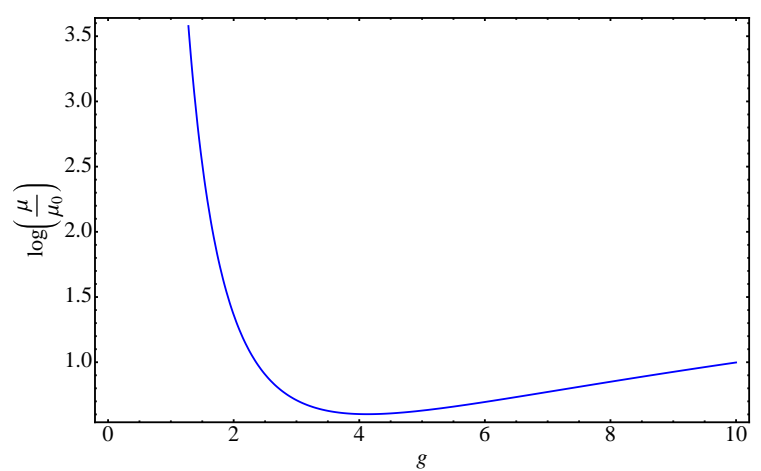

(a) $g_{0}=3$

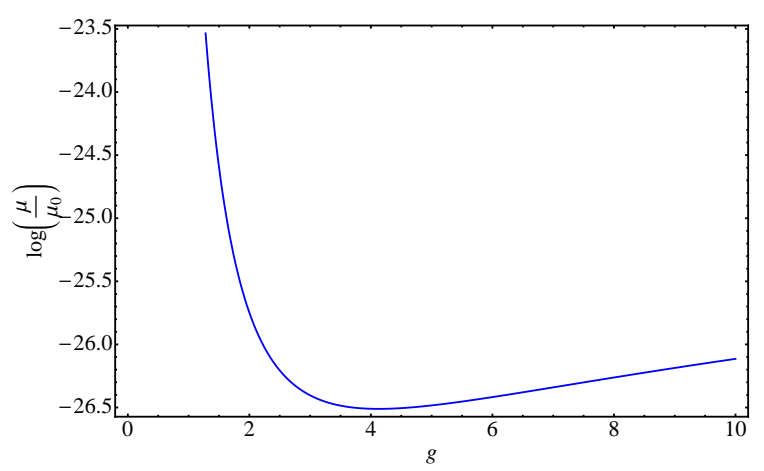

(b) $g_{0}=0.5$

FIG. 3: Solution for running scale vs. coupling constant $g$.

$$
\log (M)=\log \left(\mu_{0}\right)+\frac{1}{2 a}\left(b-\frac{1}{g_{0}^{2}}\right)-\frac{b}{a} \log \left(g_{0} b^{0.5}\right),
$$

where $g_{0}$ is the assumed value of the coupling constant at $\mu_{0}=91.19 \mathrm{GeV}$.

Note that the so defined mass gap depends on the particular N=3 Yang-Mills theory we are considering via its dependence on $g_{0}$. In Figure 4 (a) twice the mass gap, $\mathrm{M}$ in $\mathrm{GeV}$ is plotted for $g_{0}$ ranging from 1 to 10 . For orientation, the mass gap for $g_{0}$ slightly larger than one is seen to be in the $\mathrm{GeV}$ range. The mass gap reaches a maximum when $g_{0}$ takes the pole value, $1 / b^{0.5}$ and declines somewhat for larger values of $g_{0}$. It seems reasonable that within the asymptotically free regime, the mass gap increases with the strength of the coupling constant, $g_{0}$. For $g_{0}<1$, the mass gap starts to decline extremely rapidly, as may be seen from Fig. 4 (b).

To further test the reasonableness of our interpretation, we may try to predict the mass of the lightest glueball in this model. We would like the result to be similar to the usually expected value (about $1.5 \mathrm{GeV}$ ) when we adopt the QCD value for the coupling constant, $g_{0}=1.228$, mentioned above. This would correspond to neglecting the effects of quark fields on the glueball mass. Since it is seen that there is a rapid dependence of $M$ on $g_{0}$ we give in Fig. 5 a blow up of the prediction for $M / 2$ in the region of $g_{0}$ near the experimental one. This yields for the lightest glueball mass,

$$
M / 2 \approx 1.67 \mathrm{GeV},
$$

which does seem reasonable for an a priori prediction. We may compare this value with the result of a lattice QCD calculation employing a valence assumption [8]. That calculation gave the result for the $0^{+}$glueball which is found to be lightest:

$$
m\left(0^{+}\right)=1648 \pm 58 \mathrm{MeV} .
$$

This embarassingly accurate agreement gives us at least some confidence in the correctness of the interpretation of the mass gap and the validity of the RS conjectured beta function. Other lattice results are also in agreement with the value quoted above [9, 10]. To sum up, the pole in the beta function does not produce any singularity in the theory but seems to be the feature which generates the mass gap. It would be interesting to investigate the dependence of this result on the choice of renormalization scheme.

If the conjectured pure Yang-Mills beta function could be shown to be the same as the exact one, it would amount to showing that the theory has the kind of mass gap defined above.

Actually, it is easy to see that this mass gap mechanism does not, in general, require the RS conjecture if one

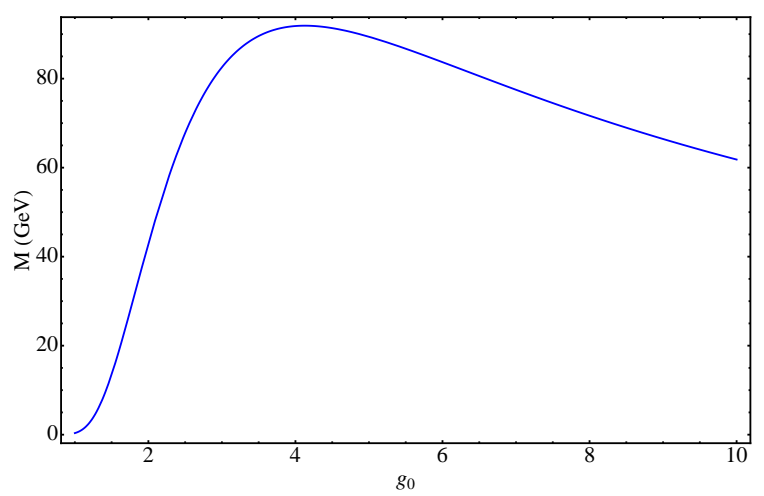

(a) $M$ (twice the mass gap) dependence on $1 \leq g_{0} \leq 10$

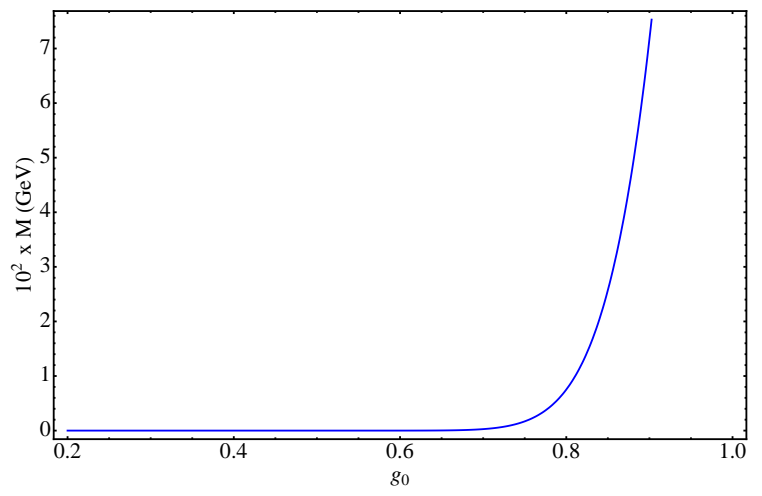

(b) $M$ (twice the mass gap) dependence on $g_{0} \leq 1$

FIG. 4: $M$ (twice the mass gap) dependence on $g_{0}$ for $\mu_{0}=$ 91.19 $\mathrm{GeV}$ and $g=b^{-0.5}$. 


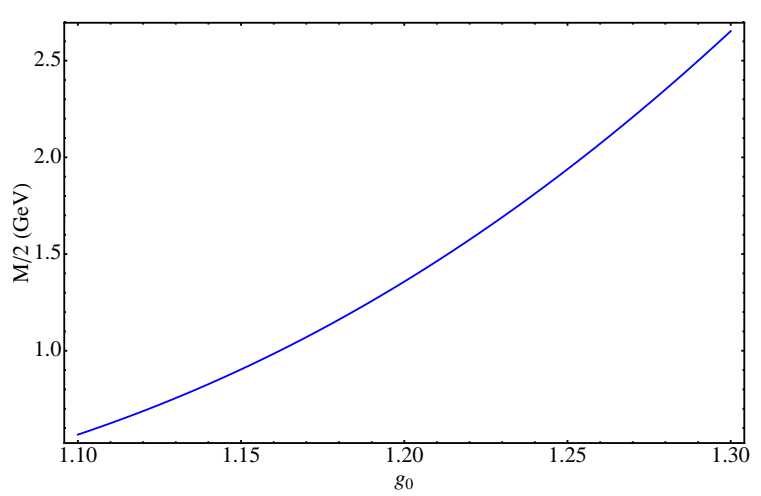

FIG. 5: Mass gap dependence on $1.1 \leq g_{0} \leq 1.3$ for $\mu_{0}=$ 91.19 $\mathrm{GeV}$ and $g=b^{-0.5}$.

goes beyond the ordinary Yang-Mills model. That is because the supersymmetric Yang-Mills theory, which is of course the starting point of the RS conjecture, is known to possess an exact beta function of the same form as Eq.(4) but with somewhat different values of $a$ and $b$, namely:

$$
a^{\prime}=\frac{3 N}{(4 \pi)^{2}}, \quad b^{\prime}=\frac{N}{8 \pi^{2}}
$$

The numerical results are qualitatively similar if one assumes a similar value of the coupling constant. For example if we choose to specify the super Yang-Mills theory by taking a similar coupling constant at the scale, $\mu_{0}=m_{Z}$, we would get for the choice $g_{0}=1.0$, the curve shown in Fig. 6. which describes the running of $g(\mu)$ with respect to $\log (\mu)$ and can be compared with the curve in Fig. 2(b).

We can compare the mass gap, $M^{\prime} / 2$ in the supersymmetric case by using Eq. (10) and making the choice $g_{0}=$ 1.228 (and also $\mathrm{N}=3$ ). This would give for the mass of the lightest supersymmetric multiplet,

$$
M^{\prime} / 2 \approx 0.49 \mathrm{GeV}
$$

Clearly, the supersymmetric and non supersymmetric Yang-Mills model results seem to be only qualitatively similar, the lightest supersymmetric particle having a mass about $1 / 3$ that of the non-supersymmetric model glueball (assuming the same coupling constant).

Of course, another interesting aspect to explore for gauge theories is their behavior as the number of colors, $\mathrm{N}$ gets large. Taking the supersymmetric gauge theory as an example and fixing, for the sake of definiteness, the gauge coupling constant as 1.228 at $\mu_{0}=91.19 \mathrm{GeV}$, we find the running of $g(\mu)$ by substituting Eq.13) into Eq.99. Figure 7 (a) show $\log (\mu)$ plotted against $g(\mu)$ for respectively $\mathrm{N}=3$ (blue solid line), $\mathrm{N}=15$ (red dashed line) and $\mathrm{N}=100$ (black dot-dashed line). It is seen that the asymptotically free (left) region in $g$ shrinks as $\mathrm{N}$ increases. Furthermore, the mass gap (corresponding to

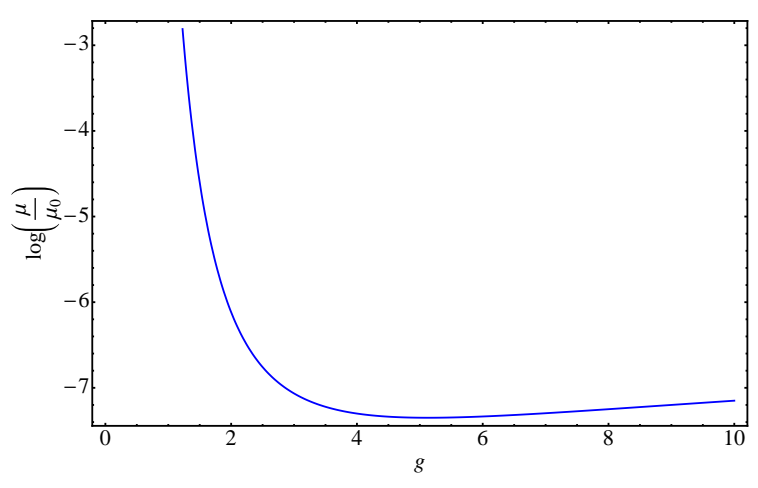

FIG. 6: Solution of the running of the scale as function of the coupling constant $g$ for the Super Yang-Mills theory.

the ordinate at the minimum point) is shown in Fig. 7 (b). It shows that the mass gap has a maximum as function of the number of colors.

When considering the large $\mathrm{N}$ behavior of gauge theories it is often desired to make an extrapolation where large $\mathrm{N}$ is taken in such a way that the quantity,

$$
g_{t}^{2} \equiv g^{2} N
$$

is held fixed. With such an extrapolation there will be no dependence (in either the supersymmetric YangMills or the pure Yang-Mills with RS type beta function cases) on $\mathrm{N}$. This may be immediately seen by writing, from Eq. (4), $a \equiv \tilde{a} N$ and $b \equiv \tilde{b} N$ so that $\tilde{a}$ and $\tilde{b}$ are independent of N. Then, as an example, Eq.(10) for the mass gap becomes

$$
\log \left(\frac{M}{\mu_{0}}\right)=\frac{\tilde{b}}{\tilde{a}}\left(\frac{1}{2}-\log \left(\tilde{b}^{1 / 2} g_{0} N^{1 / 2}\right)\right)-\frac{1}{2 \tilde{a} g_{0}^{2} N}
$$

which only involves $\mathrm{N}$ via the fixed combination $g_{0}^{2} \mathrm{~N}$.

\section{SUMMARY AND DISCUSSION}

First, an exploration of the scale dependence of physical quantities (with engineering dimension, $D$ ) was made using dimensional analysis and a specific form, conjectured by Ryttov and Sannino, of the beta function for the pure Yang-Mills theory. It was noted that even though the beta function had a pole (inherited from the known form for supersymmetric gauge theories which stimulated the conjecture) physical quantities remained smooth as the pole value of the coupling constant, $g$ was crossed.

For a more detailed understanding of how the coupling constant runs, the integration of the beta function was next carried out for the complete set of SU(3) YangMills theories, i.e. those corresponding to any choice of coupling constant, $g_{0}$ at a convenient reference scale, 


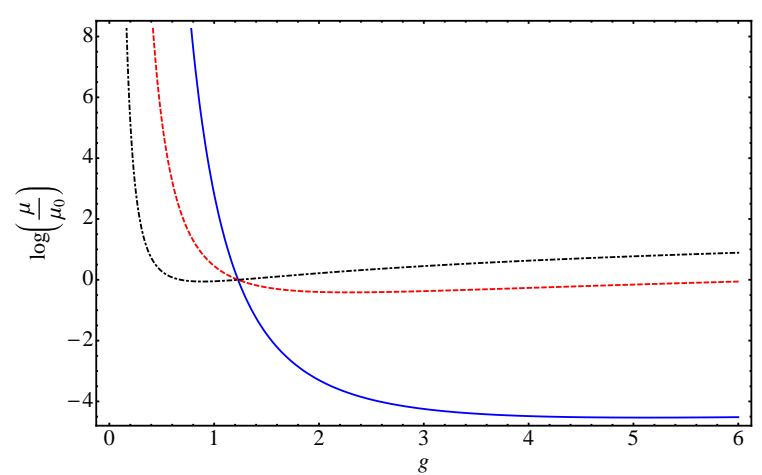

(a) Running of the scale as function of $g$ for Super Yang-Mills.

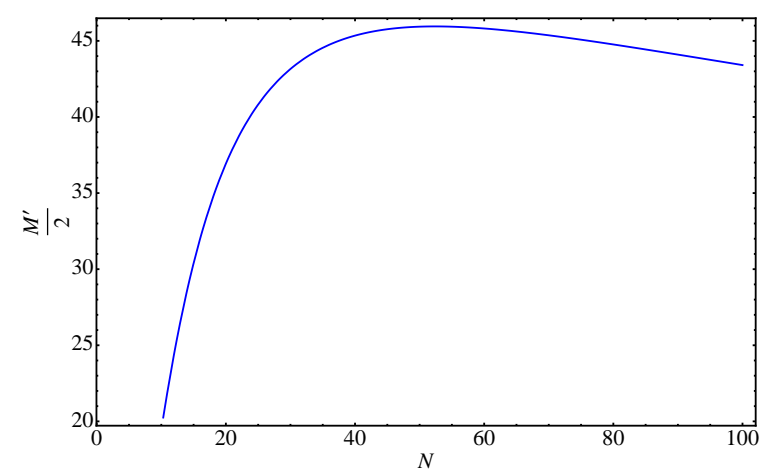

(b) Mass gap dependence on $N$ for Super Yang-Mills

FIG. 7: In the panel (a) we plot the solution of the running of the scale as function of the coupling constant $g$ for the Super Yang-Mills theory for $N=3$, solid blue line; $N=15$, red-dashed line and $N=100$, black-dot-dashed line. We have kept fixed $g_{0}=1.228$ at the scale $\mu_{0}=91.19 \mathrm{GeV}$. In the panel (b) we plot the mass gap $M^{\prime} / 2$ as function of the number of colors $N$.

$\mu_{0}=m_{Z}$. Then $g(\mu)$ was also seen to have a smooth behavior at the pole value of $g$. Most interesting is that (due to the existence of the pole) the curve of $g(\mu)$ predicts a numerical value for the mass gap of the YangMills theory, i.e. the mass of the lightest glueball. The predicted value, $1.67 \mathrm{GeV}$, seems rather close to the one obtained from a lattice treatment of QCD in the valence or quenched approximation.

It is noted that if the reference value of the coupling constant, $g_{0}$ decreases below about 1 (the experimental value is 1.228) the mass gap drops very quickly.

A similar treatment was carried out for the supersymmetric SU(3) Yang-Mills theory and the mass gap was calculated to be about $0.49 \mathrm{GeV}$. In this case, the form of the beta function is quite similar but is based on a known rather than a conjectured beta function.
We plan to next investigate the extent to which this work can be carried out for non-supersymmetric gauge theories containing fermions. Evidently there are many interesting questions which remain.

\section{Acknowledgments}

We would like to thank Claudio Pica for useful discussions and comments. The work of JS was supported in part by the US DOE under Contract No. DE-FGG-0285ER 40231; he would also like to thank the $\mathrm{CP}^{3}$-Origins group at the University of Southern Denmark, where this work was started, for their warm hospitality and partial support.
[1] S. Adler and W. Bardeen, Phys. Rev. 183, 1517 (1969).

[2] S. Ferrara and B. Zumino, Nucl. Phys. B87, 207 (1975).

[3] D.R.T. Jones and J.P. Leveille, Phys. Lett. 109B, 449 (1982); Nucl. Phys. B206, 473 (1982); D.R.T. Jones Phys. Lett. 123B,45 (1983).

[4] V.A. Novikov, M.A. Shifman, A.I. Vainshtein and V.I. Zakharov, Nucl. Phys. B229, 381 (1983);M.A. Shifman, A.I. Vainshtein, Nucl. Phys. B277, 456 (1986).

[5] T. A. Ryttov and F. Sannino, Phys. Rev. D 78, 065001 (2008) arXiv:0711.3745 [hep-th]].
[6] F. Sannino, Phys. Rev. D 79, 096007 (2009) |arXiv:0902.3494 [hep-ph]].

[7] F. Sannino, Acta Phys. Polon. B 40, 3533 (2009) |arXiv:0911.0931[hep-ph]].

[8] A. Vaccarino and D. Weingarten, Phys. Rev. D 60, 114501 (1999).

[9] C. J. Morningstar and M. J. Peardon, Phys. Rev. D 56 (1997) 4043 |arXiv:hep-lat/9704011|.

[10] B. Lucini, A. Rago and E. Rinaldi, arXiv:1007.3879 [heplat]. 